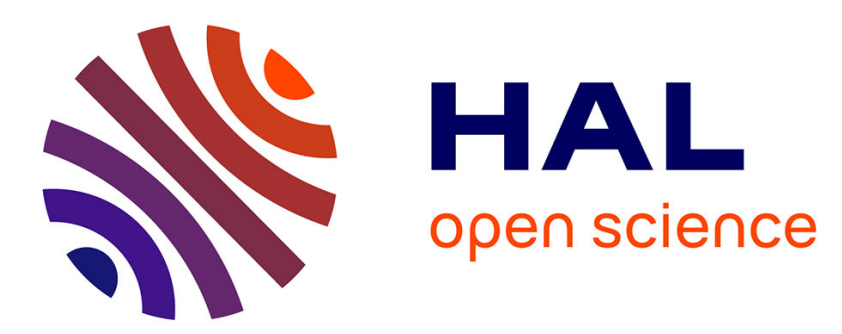

\title{
Etude comparative de quelques méthodes d'estimation de l'aptitude à la compétition saprophytique dans le sol des Trichoderma
}

Pierre Davet, Pierre Camporota, Christine Roure

\section{- To cite this version:}

Pierre Davet, Pierre Camporota, Christine Roure. Etude comparative de quelques méthodes d'estimation de l'aptitude à la compétition saprophytique dans le sol des Trichoderma. Agronomie, 1986, 6 (6), pp.575-581. hal-00884912

\section{HAL Id: hal-00884912 \\ https://hal.science/hal-00884912}

Submitted on 1 Jan 1986

HAL is a multi-disciplinary open access archive for the deposit and dissemination of scientific research documents, whether they are published or not. The documents may come from teaching and research institutions in France or abroad, or from public or private research centers.
L'archive ouverte pluridisciplinaire HAL, est destinée au dépôt et à la diffusion de documents scientifiques de niveau recherche, publiés ou non, émanant des établissements d'enseignement et de recherche français ou étrangers, des laboratoires publics ou privés. 


\title{
Etude comparative de quelques méthodes d'estimation de l'aptitude à la compétition saprophytique dans le sol des Trichoderma
}

\author{
Pierre DAVET \& Pierre CAMPOROTA (*) \\ avec la collaboration technique de Christine RourE
}

I.N.R.A., Laboratoire de Biologie et Pathologie végétales de l'ENSAM, F 34060 Montpellier Cedex

(*) I.N.R.A., Station de Recherches sur la Flore pathogène dans le Sol, 17, rue Sully, F 21034 Dijon Cedex

\begin{abstract}
On compare les avantages et les inconvénients de quatre méthodes d'appréciation de l'aptitude à la compétition saprophytique de 20 clones de Trichoderma. La détermination de l'activité cellulasique, qui correspond à un caractère pour lequel on sait que des travaux de sélection sont possibles, ne rend que partiellement compte du comportement saprophytique des clones. La colonisation de fragments de paille dans le sol constitue un modèle proche des conditions naturelles, mais la forte variabilité des résultats rend son emploi peu aisé. La méthode des pastilles gélosées, assez artificielle, est facile à mettre en œuvre et sa reproductibilité est satisfaisante. Elle permet une bonne discrimination entre les clones. La méthode des pièges de papier filtre est bien adaptée à des études de dynamique des populations. Les résultats de ces quatre méthodes d'appréciation sont convergents et permettent de distinguer des clones très compétitifs et des clones pourvus d'une faible aptitude à la compétition saprophytique dans les conditions de cette étude.
\end{abstract}

Mots clés additionnels : Clones, tri, activité cellulasique.

Additional key words : Clones, screening, cellulase activity.

\section{INTRODUCTION}

Les Trichoderma, en particulier T. hamatum (Bon.) Bain. et $T$. harzianum Rifai, sont des agents possibles de lutte biologique contre des champignons parasites telluriques (PAPAVIZAS \& LUMSDEN, 1980 ; DAVET, 1983), à condition toutefois que leurs qualités d'antagonistes puissent s'exprimer dans le milieu microbiologiquement surpeuplé que constituent les sols cultivés.
Pour être efficace, un clone de Trichoderma doit en effet posséder non seulement une activité mycoparasitaire spécifique, mais aussi une bonne aptitude à la compétition saprophytique. Pour choisir judicieusement les souches, il faut pouvoir apprécier ce caractère d'une manière rapide et sûre. En dehors des travaux de GARRETT et de son école, à Cambridge (GARRETT, 1950 ; BUTLER, 1953 ; LUCAS, 1955 ; RAO, 1959) et de quelques autres (BARTON, 1961 ; PAPAVI- 
ZAS \& DAVEY, 1961 ; DAVET, 1976 ; CAMPOROTA, 1981), il ne semble pas que les chercheurs aient accordé beaucoup d'attention à l'aptitude des champignons telluriques à coloniser un substrat en situation de compétition. C'est pourquoi nous avons entrepris une étude méthodologique qui nous a amenés à comparer 4 techniques d'évaluation de la compétitivité des clones de Trichoderma en présence de la microflore du sol. L'une est une méthode enzymatique; les autres s'inspirent plus ou moins de la méthode de Cambridge.

\section{MATÉRIEL ET MÉTHODES}

\section{A. Matériel cryptogamique}

Nous comparons un ensemble de 20 isolements monoconidiens de Trichoderma, désignés par la suite comme clones. Ces clones font partie d'une collection décrite précédemment (ARTIGUES \& DAVET, 1984) et conservée à $5{ }^{\circ} \mathrm{C}$ sur milieu à la pomme de terre (PDA). Certains essais sont conduits avec des sousensembles de 10 à 12 de ces clones.

\section{B. Sol}

La terre utilisée dans tous les essais provient d'un jardin de Montpellier. C'est un sol sablo-limoneux de $\mathrm{pH}\left(\mathrm{CaCl}_{2}\right)=6,7$ contenant 1,94 p. 100 de carbone organique.

\section{Multiplication des Trichoderma}

- Pour les essais de colonisation saprophytique, les clones sont ensemencés dans des fioles de Roux sur un milieu constitué d'un mélange de sable et de farine d'avoine, humidifié ( $200 \mathrm{~g}$ de sable, $6 \mathrm{~g}$ de farine d'avoine et $30 \mathrm{ml}$ d'eau distillée ; 2 autoclavages à $24 \mathrm{~h}$ d'intervalle). Ces cultures sont maintenues à $28{ }^{\circ} \mathrm{C}$ pendant $12 \mathrm{j}$, puis elles sont laissées pendant une période équivalente dans les conditions du laboratoire et à la lumière. Les fioles sont alors vidées, leur contenu est homogénéisé puis séché à l'air dans des conditions aseptiques pendant 2 à $3 \mathrm{j}$. Les poudres ainsi obtenues sont conservées à $5{ }^{\circ} \mathrm{C}$ dans des boîtes étanches et constituent ce que nous appellerons par la suite l'inoculum.

- Pour les essais enzymatiques, les clones sont cultivés en milieu liquide dans des fioles de Roux contenant un disque de filtre cellulosique de $12,5 \mathrm{~cm}$ de diamètre, découpé en 8 secteurs égaux, et $80 \mathrm{ml}$ de solution minérale (pour $1 \mathrm{l}: \mathrm{Ca}\left(\mathrm{NO}_{3}\right)_{2}, 1 \mathrm{~g} ; \mathrm{KNO}_{3}$, $250 \mathrm{mg} ; \mathrm{MgSO}_{4}, 7 \mathrm{H}_{2} \mathrm{O}, 250 \mathrm{mg} ; \mathrm{KH}_{2} \mathrm{PO}_{4}, 125 \mathrm{mg}$; $\mathrm{K}_{2} \mathrm{HPO}_{4}, 125 \mathrm{mg}$ ). Les flacons sont mis en incubation pendant $9 \mathrm{j}$ à $28{ }^{\circ} \mathrm{C}$.

\section{Colonisation saprophytique de la paille}

L'inoculum est mélangé à la terre tamisée à raison de 0,1 p. 100 (vol./vol.). En pratique, cette propor- tion est obtenue par pesée, en tenant compte des densités apparentes de la terre et de l'inoculum. Le mélange est homogénéisé à sec pendant $8 \mathrm{mn}$ dans un homogénéiseur mécanique (Turbula). A $200 \mathrm{ml}$ de sol ainsi inoculé, on ajoute $2 \mathrm{~g}$ de paille de blé hachée en fragments de $2 \mathrm{~cm}$ environ, puis de l'eau de façon à atteindre 60 à 70 p. 100 de la capacité de rétention. Après brassage à l'aide d'une cuillère, l'ensemble est introduit dans un sac de polyéthylène dont l'ouverture est scellée avec un ruban adhésif. Les sacs sont mis en incubation à l'étuve à $28^{\circ} \mathrm{C}$, à l'obscurité. Quatre jours plus tard, la paille est recueillie, lavée sous un courant d'eau, désinfectée superficiellement à l'hypochlorite de sodium pendant $2 \mathrm{mn}$, rincée et découpée en fragments de $5 \mathrm{~mm}$ de long. Pour chaque sac, 100 de ces fragments sont mis en culture sur un milieu sélectif pour les Trichoderma (DAVET, 1979). Après une semaine d'incubation à $28^{\circ} \mathrm{C}$, on compte le nombre de fragments de paille colonisés. Pour chaque clone, il est fait au moins 4 répétitions.

\section{E. Estimation du développement des Trichoderma en compétition par la méthode des pastilles gélosées}

\section{Principe}

Cette méthode consiste à mélanger des proportions croissantes d'inoculum à de la terre. Un échantillon de $12 \mathrm{~g}$ de chaque mélange est réparti au fond d'une boîte de Petri. On verse par-dessus $20 \mathrm{ml}$ d'eau gélosée en surfusion et l'on imprime à la boîte un mouvement circulaire rapide de façon à bien homogénéiser l'ensemble. Après refroidissement et solidification, des pastilles de $8 \mathrm{~mm}$ de diamètre sont découpées à l'emporte-pièce et déposées sur du milieu PDA, à raison de 4 , disposées en croix, par boîte de Petri. Les boîtes sont mises en incubation à l'obscurité à 22 ou $28{ }^{\circ} \mathrm{C}$ pendant $5 \mathrm{j}$ puis restent $2 \mathrm{j}$ à la lumière à la température du laboratoire avant d'être notées. Cinq boîtes de 4 pastilles sont ensemencées pour chaque traitement. Les essais sont répétés 2 à 5 fois, selon les séries.

\section{Gammes de concentrations}

Dans une $1^{\text {re }}$ série d'essais portant sur toute la collection, on a mélangé à la terre des quantités d'inoculum identiques quels que soient les clones, dans des proportions de 6, 24 et 90 p. 100 en poids, sans tenir compte de leur richesse réelle en propagules.

Dans une $2^{e}$ série, on a d'abord évalué la teneur en propagules de chaque inoculum, selon la technique de DAVET (1979), puis on a mélangé à la terre des quantités ajustées de façon à avoir les mêmes teneurs en propagules pour chaque clone. Nous avons utilise pour ces essais une douzaine de clones seulement, ce qui nous a permis d'améliorer la précision en travaillant avec 4 taux d'inoculum au lieu de 3 , à savoir $63000,316000,912000$ et $24 \times 10^{6}$ propagules $/ g$.

\section{Notation}

Chaque pastille est notée de 0 (aucun développement) à 4 (toute la surface autour de la pastille est colonisée par le Trichoderma). Les notes des 20 pastilles correspondant à chaque traitement sont ensuite 
additionnées, la note maximum étant donc 80 . Il est alors possible de calculer la proportion théorique d'inoculum (ou la quantité de propagules/g de sol) nécessaire pour obtenir la note 40 , moitié de la note maximum. Ces valeurs, appelées $C 40$, peuvent être déterminées graphiquement, en construisant les courbes représentant les notes obtenues en fonction des concentrations. On peut aussi linéariser les courbes, après avoir exprimé les concentrations en logarithmes et obtenir par le calcul les valeurs recherchées. Dans les 2 cas, on aboutit à un classement des clones, les plus compétitifs étant ceux dont la valeur de C 40 est la plus basse. On peut encore classer les clones d'une $3^{\mathrm{e}}$ manière, en attribuant à chacun d'eux une note égale au total des différentes notes obtenues pour chacune des concentrations essayées.

\section{F. Estimation du développement saprophytique en compétition par la méthode des pièges de papier- filtre}

\section{Principe}

La méthode a été décrite en détail antérieurement (CAMPOROTA, 1983). Elle consiste à enfouir dans le sol, humidifié à 70 p. 100 de sa capacité de rétention, des pièges constitués de rondelles de papier filtre de $10 \mathrm{~mm}$ de diamètre, imprégnées de milieu de RICHARDS (pour $1 \mathrm{l}: \mathrm{KH}_{2} \mathrm{PO}_{4}, 1 \mathrm{~g} ; \mathrm{MgSO}_{4}, 7 \mathrm{H}_{2} \mathrm{O}$, $0,5 \mathrm{~g} ; \mathrm{FeSO}_{4}, 7 \mathrm{H}_{2} \mathrm{O}, 5 \mathrm{mg} ; \mathrm{ZnSO}_{4}, 5 \mathrm{mg} ; \mathrm{MnCl}_{2}$, $2 \mathrm{mg}$; galactose, $20 \mathrm{~g}$; glycocolle, $2,25 \mathrm{~g}$ ) additionné de fongicides (rose bengale, $100 \mathrm{mg}$; sulfate de streptomycine, $100 \mathrm{mg}$; bénomyl, $5 \mathrm{mg}$; sulfate de cuivre, $5 \mathrm{mg}$; prothiocarbe, $50 \mathrm{mg}$; pentachloronitrobenzène, $50 \mathrm{mg}$ ). Pour chacune des concentrations, on remplit 4 boîtes de Petri de $90 \mathrm{~mm}$ de diamètre avec une quantité constante de mélange dans lequel, après humidification, on place verticalement 10 pièges par boîte, espacés de $20 \mathrm{~mm}$ en tous sens. Après une incubation de $5 \mathrm{j}$ à $25^{\circ} \mathrm{C}$, ces pièges sont rincés, essorés et déposés sur le milieu sélectif de DAVET (1979). La colonisation des pièges par les Trichoderma est notée après $48 \mathrm{~h}$ d'incubation à $25^{\circ} \mathrm{C}$.

\section{Quantification}

Le sol analysé est dilué par mélange avec de la terre de serre désinfectée à 3 reprises à $100{ }^{\circ} \mathrm{C}$, de façon à avoir des concentrations de $100,10,1,0,1$ et 0,01 p. 100. On calcule la droite de régression liant le pourcentage de colonisation des pièges et les concentrations correspondantes. On résout ensuite l'équation de la droite pour une colonisation de 50 p. 100 : ceci permet de déterminer le poids de sol nécessaire pour que 50 p. 100 des pièges soient colonisés dans les conditions du test. Ce poids est appelé « Unité de Colonisation 50 » (UC 50) que l'on exprime, afin de pouvoir comparer différents échantillons de sol entre eux, en nombre d'UC $50 / \mathrm{g}$ de terre.

\section{Protocole expérimental}

On compare le comportement saprophytique, dans le sol de Montpellier, de 10 des clones de la collection. Deux dates d'analyse ont été retenues : au moment de l'introduction de l'inoculum dans le sol ( $\mathrm{J} 0)$ et après
$30 \mathrm{j}$ d'incubation du sol inoculé ( $\mathrm{J}$ 30). La richesse en propagules de l'inoculum produit sur sable est déterminée ici après étalement de $10 \mathrm{mg}$ dans 10 boîtes de Petri contenant du milieu au malt gélosé, en 4 répétitions. Le sol est inoculé à raison de 15 «grains fertiles $\gg / g$ et constitue le stock initial qui sera ensuite dilué avec de la terre stérile. Après humidification, chaque lot est divisé en 2 parties : l'une est analysée aussitôt, l'autre est conservée à $28{ }^{\circ} \mathrm{C}$ dans un cristallisoir fermé par un film plastique perforé pour être analysée après $30 \mathrm{j}$.

\section{G. Hydrolyse de la carboxyméthylcellulose}

Les cultures en milieu liquide sont filtrées sur de la mousseline, puis sur du papier filtre, et le filtrat est immédiatement utilisé. L'activité enzymatique est appréciée par la méthode colorimétrique de NELSONSOMOGYI (THOMAS \& REYMOND, 1958). Le milieu réactionnel est constitué d'1 $\mathrm{ml}$ de filtrat et d' $1 \mathrm{ml}$ de solution de carboxyméthylcellulose à 1,2 p. 100 dans un tampon citrate $0,05 \mathrm{M}$ à $\mathrm{pH}=4$. Le dosage du glucose libéré est réalisé après $6 \mathrm{~h}$ d'incubation à $38{ }^{\circ} \mathrm{C}$. Chaque analyse est répétée au moin 4 fois.

\section{H. Calculs}

Les analyses de corrélation sont faites par la méthode des corrélations de rangs de SPEARMAN.

\section{RÉSULTATS}

\section{A. Colonisation de la paille}

Le temps relativement court pendant lequel les fragments de paille restent enfouis dans le sol permet une bonne discrimination des clones, malgré une variabilité assez forte d'une répétition à une autre (tabl. 1). On peut ainsi distinguer des clones ayant une très faible activité saprophytique (taux de colonisation inférieurs à 5 p. $100:$ LN3, JI1, LN1) et des clones ayant une activité élevée (taux de colonisation supérieurs à 50 p. 100 : HH1, MB, 24).

\section{B. Activité saprophytique estimée par la méthode des pastilles gélosées}

La reproductibilité de la méthode est bonne comme le montre l'exemple de la figure 1. Nous avons comparé les classements issus des 3 procédés d'interprétation des résultats décrits au chapitre précédent : détermination graphique de C 40 , calcul de C 40 après linéarisation, classement selon le total des notes (tabl. 2). On peut les considérer comme identiques, les coefficients de corrélation entre classements étant compris entre 0,97 et 0,99 . Nous avons comparé aussi les 2 méthodes de préparation des mélanges : apport d'inoculum en proportion déterminée, sans tenir 
TABLEAU 1

Pourcentage moyen de fragments de paille colonisés par chaque clone, après $4 j$ d'incubation dans un sol non stérile, à $28^{\circ} \mathrm{C}$.

Mean percentage of straw pieces colonized by each clone, after a 4-day incubation at $28^{\circ} \mathrm{C}$ in non-sterile soil.

\begin{tabular}{lcrc}
\hline \hline Clone & $\begin{array}{c}\text { Pourcentage de } \\
\text { fragments colonisés }\end{array}$ & Ecart-type & Classement \\
\hline HH1 & 61,8 & 21,17 & 1 \\
MB & 57,0 & 24,02 & 2 \\
24 & 50,5 & 28,23 & 3 \\
HH3 & 50,0 & 27,88 & 4 \\
B140 & 45,7 & 27,87 & 5 \\
B1 & 44,8 & 24,10 & 6 \\
V1 & 43,8 & 19,48 & 7 \\
3160 & 43,0 & 28,77 & 8 \\
LR3 & 38,0 & 20,41 & 9 \\
LK1 & 31,8 & 19,31 & 10 \\
LX3 & 16,5 & 7,94 & 11 \\
LX1 & 16,2 & 9,04 & 12 \\
LR1 & 16,0 & 8,22 & 13 \\
LW1 & 14,3 & 11,95 & 14 \\
JE1 & 11,8 & 10,14 & 15 \\
KZ1 & 5,8 & 3,27 & 16 \\
MD1 & 5,2 & 7,94 & 17 \\
LN1 & 3,5 & 1,00 & 18 \\
JI1 & 2,8 & 4,27 & 19 \\
LN3 & 2,5 & 1,29 & 20 \\
\hline \hline
\end{tabular}

compte de la teneur en propagules, et ajustement des quantités d'inoculum de façon à travailler avec des taux de propagules identiques pour tous les clones. $\mathrm{La}$ correspondance entre les classements obtenus par ces 2 méthodes est très bonne : $r=0,95$.

Dans tous les cas, on observe des différences importantes dans le comportement des clones : alors qu'il suffit d'un faible apport des clones 24 ou B1 pour atteindre la note 40 , il faut au contraire des quantités très importantes d'inoculum de JI1, LN1 ou LN3 pour obtenir le même résultat.

La température d'incubation $\left(22\right.$ ou $\left.28{ }^{\circ} \mathrm{C}\right)$ modifie peu le classement général des clones (tabl. 3). On peut cependant noter que les 2 clones les plus compétitifs à $22{ }^{\circ} \mathrm{C}$ ont une température optimale de croissance de $22-24{ }^{\circ} \mathrm{C}$, alors que le clone le mieux classé à $28^{\circ} \mathrm{C}$ a aussi son optimum thermique à $28^{\circ} \mathrm{C}$.

\section{Activité saprophytique estimée par la méthode des pièges de papier-filtre}

A J0, les estimations des UC 50/g s'échelonnent de 0,1 à 100 selon les clones (fig. 2). On peut distinguer approximativement 2 groupes: HH3, B1, 24, 3160, MB et HH1 sont des clones montrant dans cet essai une aptitude saprophytique forte à très forte, tandis que B140, LR1, LR3 et V1 se classent comme moyens à faibles. On obtient à $\mathrm{J} 30$ un classement sensiblement analogue, avec une tendance générale à la diminution du nombre d'UC $50 / \mathrm{g}$, à 3 exceptions près.

\section{Activité cellulolytique}

Les filtrats de culture hydrolysent la carboxyméthylcellulose dans des proportions variables (tabl. 4). Les clones MD1, 3160 et JE1 ont l'activité la plus faible ; B140, LR1 et MB ont l'activité la plus grande.
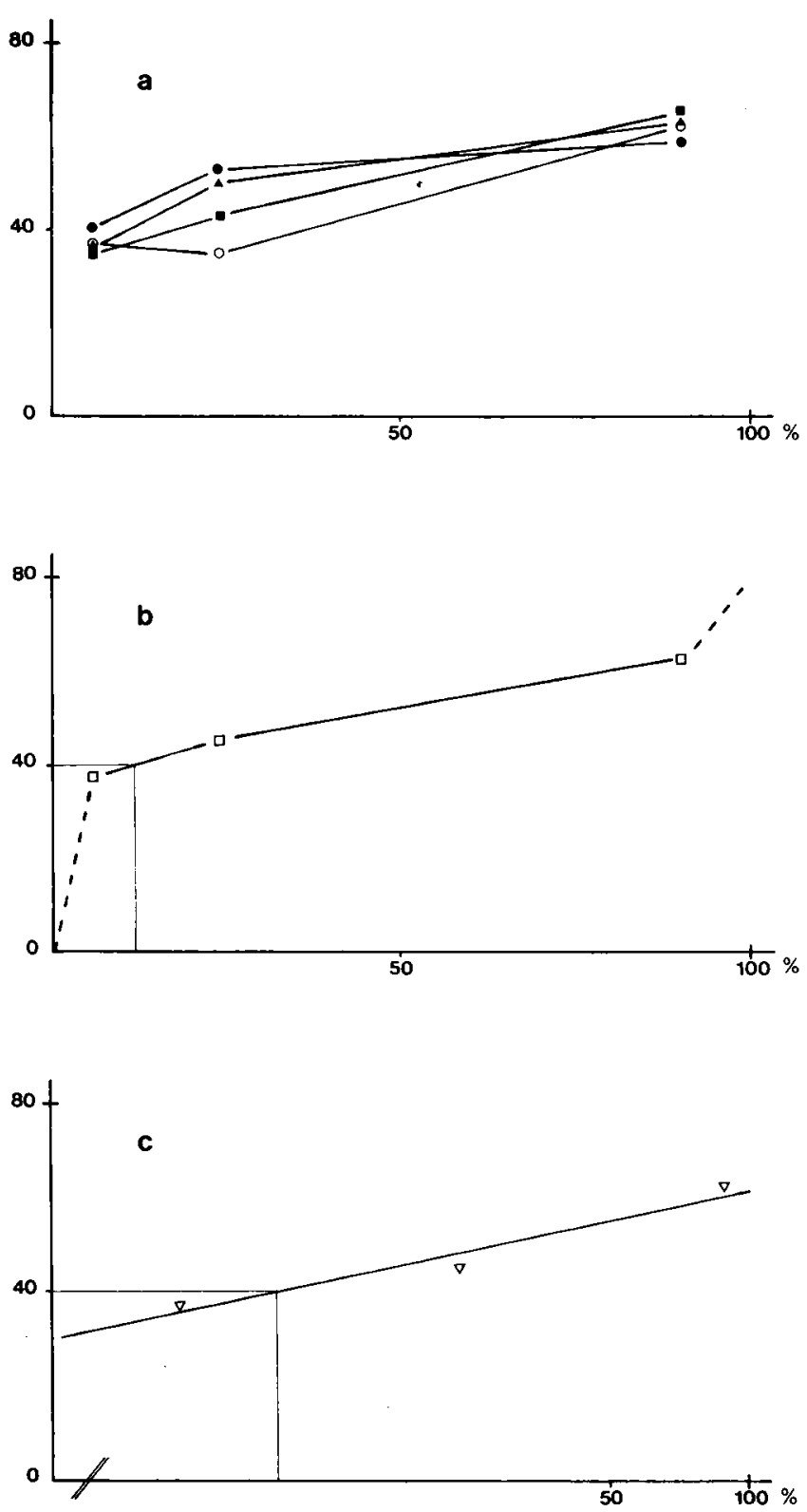

Figure 1

Exemple de résultats obtenus avec la méthode des pastilles gélosées pour le clone $\mathrm{HHI}$.

a) évolution de la notation en fonction de la proportion d'inoculum de Trichoderma, lors de 4 répétitions distinctes. L'inoculum est apporté à raison de 6, 24 et $90 \mathrm{p} .100$.

b) graphique construit à partir des moyennes des valeurs représentées sur la figure la et détermination graphique de C 40. Les parties représentées en tirets sont extrapolées en considérant que l'on obtient la note O lorsqu'il n'y a aucun inoculum et la note maximum 80 lorsqu'on a 100 p. 100 d'inoculum.

c) les points ne sont pas toujours aussi bien alignés que sur le graphique $b$ mais la courbe peut être linéarisée après expression des concentrations en logarithmes.

L'équation de la droite est ici : $y=21,22 \log x+61,63$.

Example of results obtained for clone $\mathrm{HHI}$ by the agar-disk method. a) data obtained for different proportions of Trichoderma inoculum in soil, in 4 distinct replicates. Inoculum concentrations were 6 , 24 and $90 \% \mathrm{w} / \mathrm{w}$.

b) graph constructed from the means of the values presented in fig. Ia. Parts with broken lines were extrapolated, assuming value 0 in the absence of any inoculum, and a maximum value 80 with inoculum concentration $100 \%$. The graphical determination of C 40 has also been represented.

c) dots were not always laid out in a rather straight line as in graph $b$, but the curve could be streightened if concentration was expressed logarithmically. In this case, the equation was: $y=21.22 \log x+61.63$. It was therefore easy to calculate the value of $x$ when $y=40$. 
TABLEAU 2

Exemple de résultats obtenus par la méthode des pastilles gélosées. Dans cette série d'essais, les volumes d'inoculum utilisés sont les mêmes pour tous les clones; l'incubation est faite à $28^{\circ} \mathrm{C}$. Le coefficient de corrélation entre les 2 premiers classements est 0,99 ; la corrélation entre les classements 1 et 3 est 0,$98 ;$ pour les classements 2 et $3, r=0,97$.

Example of results obtained with the agar-disk method. In the series of trials reported here, inoculum concentrations for each clone were 6,24 and $90 \%(w / w)$; the disks were incubated at $28^{\circ} \mathrm{C}$. In the first column, the clones were characterized by a graphically determined $\mathrm{C} 40$ value. In the second column, C 40 value is a theoretical number, calculated after linearization of the corresponding curves. In the third column, each clone was defined by the total of partial scores corresponding to each dilution. Correlation coefficients for classifications 1 and 2,1 and 3,2 and 3 were respectively $0.99,0.98$ and 0.97 .

\begin{tabular}{|c|c|c|c|c|c|c|}
\hline \multirow[t]{2}{*}{ Clone } & \multicolumn{2}{|c|}{$\begin{array}{l}\text { Note C } 40 \text { estimée } \\
\text { graphiquement }\end{array}$} & \multicolumn{2}{|c|}{$\begin{array}{l}\text { Note C } 40 \text { calculée } \\
\text { après linéarisation }\end{array}$} & \multicolumn{2}{|c|}{$\begin{array}{l}\text { Note obtenue par sommation } \\
\text { des notes partielles }\end{array}$} \\
\hline & C 40 & Classement 1 & C $40^{\circ}$ & Classement 2 & Note totale & Classement 3 \\
\hline 24 & 4,5 & 1 & 0,8 & 2 & 186,8 & 2 \\
\hline B1 & 5 & 2 & 1,5 & 3 & 182,2 & 3 \\
\hline 3160 & 5,5 & 3 & 0,7 & 1 & 192,4 & 1 \\
\hline HH1 & 12 & 4 & 9,5 & 4 & 144,9 & 5 \\
\hline HH3 & 14,5 & 5 & 11 & 5 & 150,2 & 4 \\
\hline MB & 21,5 & 6 & 17 & 6 & 136,0 & 6 \\
\hline V1 & 23,5 & 7 & 18 & 7 & 130,8 & 7 \\
\hline LK1 & 42,5 & 8 & 19 & 8 & 126,3 & 8 \\
\hline LR3 & 56,5 & 9 & 41 & 9 & 98,6 & 9 \\
\hline JE1 & 69,5 & 10 & 62,5 & 10 & 65,6 & 13 \\
\hline KZI & 74 & 11 & 69 & 11 & 68,2 & 12 \\
\hline LX1 & 84,5 & 12 & 102 & 12 & 77,4 & 11 \\
\hline B140 & 91 & 13 & 245 & 15 & 88,2 & 10 \\
\hline LX3 & 91 & 14 & 186 & 13 & 59,0 & 15 \\
\hline LRI & 91 & 15 & 191 & 14 & 62,8 & 14 \\
\hline LW1 & 92 & 16 & 275 & 16 & 43,3 & 16 \\
\hline MD1 & 93 & 17 & 1175 & 17 & 38,0 & 17 \\
\hline LN3 & 94 & 18 & 10000 & 18 & 16,6 & 18 \\
\hline LN1 & 95 & 19 & $468 \times 10^{12}$ & 19 & 3,9 & 19 \\
\hline JII & 95,5 & 20 & $10^{152}$ & 20 & 0,6 & 20 \\
\hline
\end{tabular}

TABLEAU 3

Comparaison, pour un échantillon de 12 clones, des classements obtenus à 22 et à $28^{\circ} \mathrm{C}$ par la méthode des pastilles gélosées (2 répétitions; nombres de propagules égaux pour chaque série de mesures).

Comparison of classifications obtained by the agar-disk method at 22 and $28^{\circ} \mathrm{C}$, with a sample of 12 clones

(means of 2 replicates; every inoculum concentration was adjusted to have the same numbers of propagules $/ g$ in each series of measurements).

\begin{tabular}{ccccccccccccc}
\hline Clone & MD1 & 24 & B1 & KZ1 & LX3 & MB & LR3 & V1 & HH1 & HH3 & LW1 & B140 \\
\hline $\begin{array}{l}\text { Température optimale } \\
\text { de croissance in vitro }\end{array}$ & 22 & 22 & 24 & 24 & 28 & 28 & 28 & 28 & 28 & 28 & 28 & 28 \\
\hline Classement à $22^{\circ} \mathrm{C}$ & 11 & 2 & 1 & 5 & 9 & 3 & 12 & 6 & 7 & 4 & 10 & 8 \\
\hline Classement à $28^{\circ} \mathrm{C}$ & 11 & 4 & 2 & 7 & 9 & 6 & 10 & 5 & 3 & 1 & 12 & 8 \\
\hline
\end{tabular}

\section{E. Correspondance entre les diverses méthodes}

Lorsqu'on estime l'activité saprophytique des Trichoderma par leur aptitude à coloniser des fragments de paille en un temps limité, on obtient un classement des clones très proche du classement établi par la méthode des pastilles gélosées $(r=0,84$, significatif à 2 p. 1000 ).

Il y a en outre une bonne corrélation entre le classement issu de la méthode des pastilles gélosées et le classement des clones établi à partir de la méthode des pièges de papier-filtre : $r=0,73$ pour les estimations à $\mathrm{J} 0$ et $\mathrm{r}=0,79$ pour les notations à $\mathrm{J} 30$. La corrélation entre le degré de colonisation des pailles et l'aptitude à dégrader la carboxyméthylcellulose est moins bonne, mais néanmoins significative au seuil de
5 p. $100(r=0,45)$. Il n'y a pas de corrélation entre cette activité enzymatique et le comportement des clones dans la méthode des pastilles gélosées, ni dans celle des pièges de papier-filtre.

\section{DISCUSSION}

Pour caractériser les clones de Trichoderma, nous avons d'abord choisi un modèle qui ne soit pas trop éloigné des conditions naturelles : la colonisation de fragments de paille enfouis dans un sol non stérile. Malgré un temps d'incubation court et une très grande dilution de l'inoculum, certains clones arrivent à 


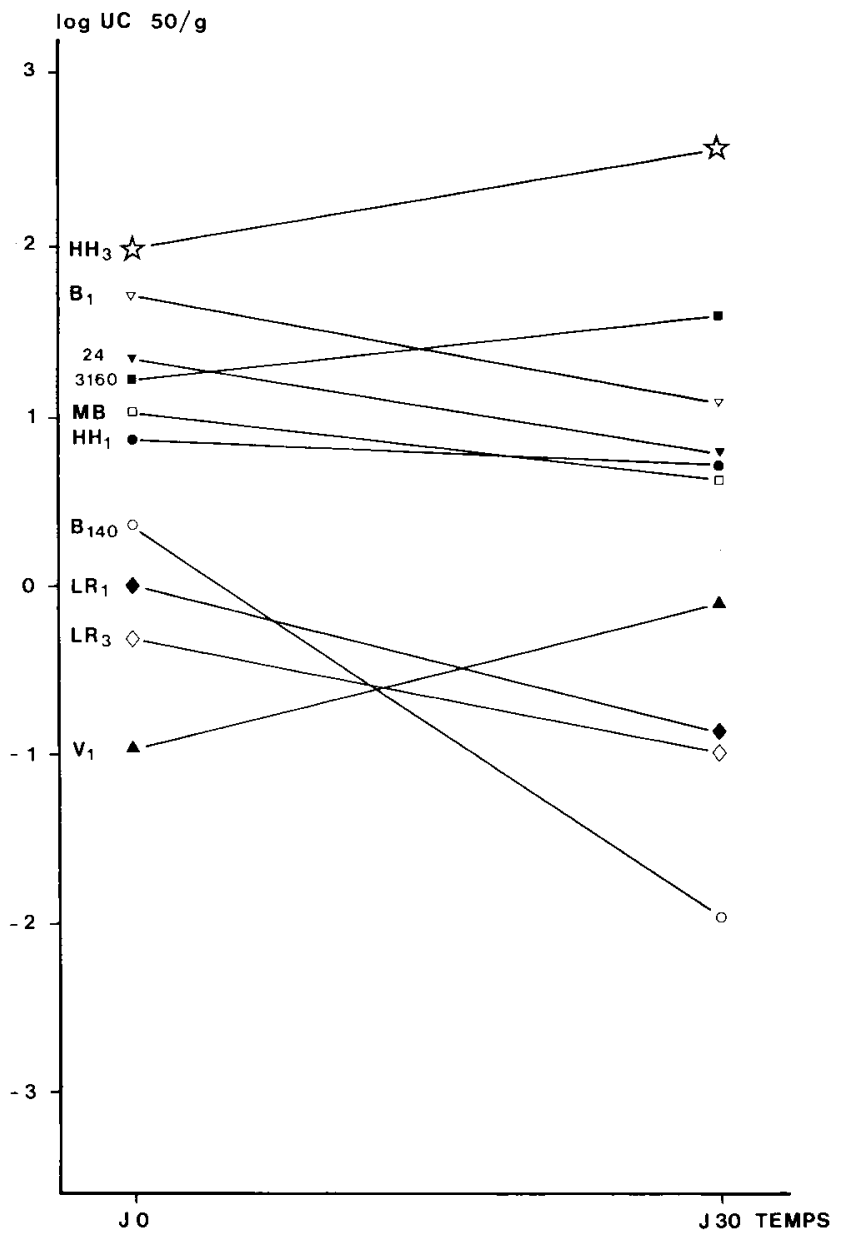

Figure 2

Méthode des pièges de papier-filtre : variation du nombre d'Unités de Colonisation 50/g de sol entre le jour de l'incorporation de l'inoculum dans la terre $(J 0)$ et la date $J 0+30$.

Filter paper trap method: the graph represents the variation of the number of Units of Colonization 50 per $\mathrm{g}$ of soil, from the day of inoculum incorporation into the soil $(J 0)$ to the date $J O+30$ days.

coloniser jusqu'à 62 p. 100 des fragments, montrant ainsi une aptitude élevée au développement saprophytique. Certains autres clones s'implantent très mal. Un travail de sélection s'impose donc puisque seuls peuvent être retenus, comme candidats à la lutte biologique dans le sol, les clones possédant une compétitivité élevée.

Nous retrouvons un résultat déjà établi par GARRETT il y a plusieurs années $(1966,1975)$ pour d'autres champignons : la capacité de coloniser les fragments de paille en situation de compétition est liée à l'activité cellulolytique des clones. Ce caractère pourrait donc servir de critère pour un travail de sélection : on sait qu'il est possible de trouver des souches de Trichoderma très fortes productrices de cellulase (MONTENECOURT \& EVELEIGH, 1977). Dans les travaux de GARRETT, l'activité cellulolytique est estimée par l'aptitude des champignons à se développer sur la cellulose du papier-filtre et la correspondance avec la colonisation des pailles est très bonne. Ici, nous avons évalué seulement l'activité du complexe enzymatique capable d'hydrolyser la carboxyméthylcellulose. Ces mesures ne prennent pas en compte l'attaque de la cellulose cristalline, ce qui explique sans doute que la corrélation observée ne soit pas très élevée, bien que significative.

L'utilisation de fragments de paille, de composition mal définie, ajoute un facteur de variabilité à ceux qui sont apportés par le sol. La méthode des pastilles gélosées et la méthode des pièges de papier-filtre permettent de travailler dans des conditions beaucoup mieux standardisées.

La méthode des pastilles gélosées a l'avantage de donner des résultats aisément reproductibles. Le substrat étant moins sélectif que la paille, on remarque, d'une part, que les doses d'inoculum nécessaires sont plus importantes puisque la concurrence est plus sévère, d'autre part, que l'activité cellulolytique n'est plus primordiale. L'activité antibiotique des Trichoderma joue certainement dans ce cas un rôle important dans leur succès pour la conquête du substrat, mais d'autres facteurs peuvent intervenir (vitesse de croissance, tolérance aux antibiotiques d'origine étrangère). Le classement auquel on aboutit est en bon accord avec le classement obtenu dans des conditions plus «naturelles" sur les fragments de paille. La technique est assez rapide et permet une bonne séparation entre clones compétitifs et clones peu actifs. L'ordre des clones n'est pas sensiblement modifié selon que l'on travaille à nombre de propagules égal ou à poids d'inoculum égal (sans tenir compte des variations éventuelles de teneur d'un inoculum à un autre). Ceci permet d'éviter le dosage préalable des propagules dans les poudres servant d'inoculum. Le classement peut être obtenu très rapidement après simple addition des notes correspondant à chacune des concentrations essayées.

La méthode des pièges de papier-filtre aboutit à un classement voisin de la méthode précédente, bien que la comparaison porte ici seulement sur la moitié des clones de la collection. Le léger écart entre les

TABLEAU 4

Activité cellulasique des clones, représentée par l'apparition de molécules de glucose dans une solution de carboxyméthylcellulose incubée pendant $6 \mathrm{~h}$ à $38{ }^{\circ} \mathrm{C}$ en présence de filtrats de culture.

Cellulolytic activity of a collection of Trichoderma strains, expressed as the weight of glucose liberated in one $\mathrm{ml}$ of carboxymethy/cellulose solution, after $6 \mathrm{~h}$ incubation at $38{ }^{\circ} \mathrm{C}$ with culture filtrates.

\begin{tabular}{cccccccccccccccccccccc}
\hline \hline Clone & B140 & LR1 & MB & HH3 & V1 & LR3 & HH1 & LX1 & LW1 & LX3 & LN3 & KZ1 & LN1 & LK1 & B1 & 24 & JI1 & MD1 & 3160 & JE1 \\
\hline $\begin{array}{l}\text { Glucose libéré } \\
\text { (mg.ml } \text { ml }^{-1}\end{array}$ & 0,67 & 0,62 & 0,61 & 0,58 & 0,53 & 0,49 & 0,45 & 0,37 & 0,32 & 0,31 & 0,29 & 0,18 & 0,17 & 0,16 & 0,16 & 0,15 & 0,11 & 0,08 & 0,08 & 0,08 \\
\hline Ecart-type & 0,10 & 0,34 & 0,14 & 0,32 & 0,32 & 0,13 & 0,15 & 0,14 & 0,16 & 0,16 & 0,21 & 0,10 & 0,16 & 0,12 & 0,15 & 0,13 & 0,11 & & \\
\hline
\end{tabular}


2 notations peut provenir du fait que les pièges utilisés sont partiellement sélectifs, ce qui modifie sensiblement les conditions de la compétition. L'intérêt de cette technique, par rapport aux précédentes, est qu'elle se prête très bien à une étude dynamique, sans exiger de grandes quantités d'inoculum et sans entraîner de perturbations du sol en cours d'étude. L'évolution du nombre d'UC $50 / \mathrm{g}$ en fonction du temps peut être considérée comme une indication de l'aptitude d'un clone à se conserver dans un sol donné.

Toutes les expérimentations rapportées ci-dessus ont été réalisées dans le même sol de Montpellier, mais les travaux en cours nous permettent de penser que nos conclusions restent valables dans d'autres types de sol. En résumé, plusieurs approches sont possibles pour apprécier l'aptitude des Trichoderma à la compétition saprophytique. Les méthodes que nous venons d'envisager donnent des résultats convergents. Pour trier des clones dans une collection, la plus facile à mettre en œuvre nous paraît être la méthode des pastilles gélosées. Pour des études de dynamique des populations, la méthode des pièges de papier filtre semble la plus intéressante.

Reçu le 19 décembre 1985. Accepté le 4 mars 1986.

\section{RÉFÉRENCES BIBL_IOGRAPHIQUES}

Artigues M., Davet P., 1984. Comparaison des aptitudes parasitaires de clones de Trichoderma vis-à-vis de quelques champignons à sclérotes. Soil Biol. Biochem., 16, 413-417.

Barton R., 1961. Saprophytic activity of Pythium mamillatum in soils. II. Factors restricting $P$. mamillatum to pioneer colonization of substrates. Trans. Br. mycol. Soc., 44, 105-118.

Butler F. C., 1953. Saprophytic behaviour of some cereal root-rot fungi. Ann. appl. Biol., 40, 284-311.

Camporota P., 1981. Mesure de la colonisation saprophytique en compétition de Rhizoctonia solani Kühn dans les sols et substrats. Agronomie, 1, 513-517.

Camporota P., 1983. Evolution du comportement saprophytique de souches de Trichoderma spp. dans les sols. Technique d'étude. Agronomie, 3, 607-609.

Davet P., 1976. Comportement sur divers substrats des champignons associés à la maladie des racines liégeuses de la tomate au Liban. Ann. Phytopathol., 8, 159-169.

Davet P., 1979. Technique pour l'analyse des populations de Trichoderma et de Gliocladium virens dans le sol. Ann. Phytopathol., 11, 529-533.

Davet P., 1983. Les Trichoderma. Exemple de champignons antagonistes d'agents pathogènes. In « Faune et flore auxiliaires en Agriculture », ACTA, Paris, 193-205.
Garrett S. D., 1950. Ecology of the root-inhabiting fungi. Biol. Rev., 25, 220-254.

Garrett S. D., 1966. Cellulose-decomposing ability of some cereal foot-rot fungi in relation to their saprophytic survival. Trans. $B r$. mycol. Soc., 49, 57-68.

Garrett S. D., 1975. Cellulolysis rate and competitive saprophytic colonization of wheat straw by foot-rot fungi. Soil Biol. Biochem., 7, 323-327.

Lucas R. L., 1955. A comparative study of Ophiobolus graminis and Fusarium culmorum saprophytic colonization of wheat straw. Ann. appl. Biol., 43, 134-143.

Montenecourt B. S., Eveleigh D. E., 1977. Preparation of mutants of Trichoderma reesei with enhanced cellulase production. Appl. Environ. Microbiol., 34, 777-782.

Papavizas G. C., Davey C. B., 1961. Saprophytic behaviour of Rhizoctonia in soil. Phytopathology, 51, 693-699.

Papavizas G. C., Lumsden R. D., 1980. Biological control of soilborne fungal propagules. Annu. Rev. Phytopathol., 18, 389-413.

Rao A. S., 1959. A comparative study of competitive saprophytic ability in twelve root-infecting fungi by an agar plate method. Trans. Br. mycol. Soc., 42, 97-111.

Thomas P., Reymond D., 1958. Techniques de Biochimie. J. B. Baillière et fils, Paris. 